\title{
DNA-Methylation-Mediated IncRNA HOXB-AS4 Promotes Gastric Cancer Progression Through Regulating miR-130a-5p/PKP4
}

\section{Xiaoyan Wang}

The Affiliated Suqian First People's Hospital of Nanjin Medical University

\section{Rong He}

The Affiliated People's Hospital of Jiangsu University

\section{Yan Wang}

The Affiliated Suqian First People's Hospital of Nanjin Medical University

\section{Yunyun Liu}

The Affiliated Suqian First People's Hospital of Nanjin Medical University

\section{Yuxin Wang}

The Affiliated Suqian First People's Hospital of Nanjin Medical University

\section{Dandan Chen}

The Affiliated Suqian First People's Hospital of Nanjin University

\section{Yu Fan ( $\nabla$ yuf12345@ujs.edu.cn )}

The Affiliated People's Hospital of Jiangsu University

\section{Research}

Keywords: Gastric cancer, HOXB-AS4, competitive endogenous RNA, DNA methylation, EMT

Posted Date: January 3rd, 2022

DOI: https://doi.org/10.21203/rs.3.rs-1202816/v1

License: (9) This work is licensed under a Creative Commons Attribution 4.0 International License. Read Full License 


\section{Abstract}

Background $\mathbb{G}$ Gastric cancer (GC) is one of the most common cancer in the world, possessing the second leading cause of cancer-related mortality. Long noncoding RNAs (IncRNAs) have been shown to play important roles in tumorigenesis. However, the effect of IncRNA HOXB-AS4 in GC progression and the underlying mechanisms remain unknown.

Methods冈Firstly, the expression of IncRNA HOXB-AS4 in gastric cancer tissues and cancer cells was investigated according to GEPIA database and Real time fluorescence quantitative PCR(qRT-PCR). Then, MTT, clone formation, Transwell and Western blot were used to study the effects of overexpression or down-regulation of HOXB-AS4 on the proliferation, invasion and epithelial mesenchymal transformation of cancer cells. We further studied the molecular mechanism of HOXB-AS4 by fluorescence in situ hybridization, bioinformatics analysis, luciferase reporting, methylation specific PCR (MSP) and chromatin immunoprecipitation (chip).

Results:In the study, the GEPIA database and quantitative Real-Time PCR (qRT-PCR) assay showed that HOXB-AS4 was upregulated in GC tissues and cells. Then, MTT, clone formation, transwell, and western blot assays suggested that overexpression of HOXB-AS4 increased cell proliferation, migration, and invasion, and regulated epithelial-mesenchymal transition (EMT) markers expression, while knockdown of HOXB-AS4 showed the opposite effect. Fluorescence in situ hybridization (FISH) assay found that HOXB-AS4 localized in the cytoplasm of the GSE-1 and AGS cells. Further mechanism experiments, including bioinformatics, luciferase reporter, qRT-PCR, and western blot assays showed that HOXB-AS4 sponged to miR-130a-5p to regulate the PKP4 expression. Knockdown of miR-130a-5p obliterated the effect of HOXB-AS4, which was further abolished by knockdown of PKP4 in vitro and in vivo. Methylationspecific PCR (MSP) and chromatin immunoprecipitation (CHIP) assay showed that overexpression of HOXB-AS4 in GC was mediated by SP1-dependent DNA methylation. Abnormal upregulation of IncRNA HOXB-AS4 contributed to GC progression, which was mediated by DNA methylation. The study clarified that DNA-methylation-mediated HOXB-AS4 played its role through miR-130a-5p/ PKP4 axis.

Conclusions: Our study provides new insights for the understanding of epigenetic regulation on IncRNA expression in GC, and indicates that HOXB-AS4 could be a biomarker of GC prognosis. Moreover, targeting HOXB-AS4 /miR-130a-5p/ PKP4 axis might be a promising strategy to treat GC.

\section{Background}

Gastric cancer (GC) is one of the most common cancer in the world, possessing the second leading cause of cancer-related mortality[1]. Although impressive progresses have been made in the treatment of GC, the survival rates of GC remain unoptimistic[2]. The survival rates of gastric correlate most with the early diagnosis, that the surgical removal of the tumor in the early stage benefits the prognosis of GC the most[3, 4]. However, the symptom of GC in the early stage may be mild and untypical, and many people were diagnosed in an advanced stage, leading to poor prognosis even then were given proper 
treatments[4]. The pathogenesis and molecular mechanism of GC are complicated, which resulted from the dysregulation of various signaling pathways[5]. So, it's of vital importance to clarify the molecular mechanism of GC progression and find new markers and targets for GC diagnosis and treatment.

Long noncoding RNAs (LncRNAs) are a group of non-coding RNAs with the length longer than 200 nucleotides[6]. LncRNA has been proved to be involved in various biological processes, including cell proliferation, differentiation, and apoptosis[7, 8]. LncRNA plays its role by regulating protein-coding genes, mRNA processing, and maintenance of genomic integrity[9]. LncRNA functioned as the competitive endogenous RNA (ceRNA) which compete with the mRNA to bind to the microRNA (miRNA), leading to the change of gene expression[10]. Although ceRNA mechanism has been reported to play roles in GC progression[11, 12], the specific molecular mechanism on the occurrence of ceRNA mechanism remains largely unknown.

Homeobox $(H O X)$ genes are a highly conserved subgroup of the superfamily, which contain 39 transcription factors[13]. It is reported that HOX family is associated with the development and progression of numerous cancers, including GC[14]. For instance, the transcription factor HOXD9 was upregulated in GC, and accelerated the GC progression[15]. Moreover, IncRNAs generated in HOX family genes have been proved to be a vital biomarker when evaluating the prognosis of multiple cancers [16, 17]. For instance, IncRNA HOXA11-AS served as an oncogene to participate in GC $[18,19]$. As is known, abnormal DNA methylation is related to many cancers, and this epigenetic disruption may lead to abnormal IncRNA expression in tumor tissues [20]. Evidence has shown that IncRNAs generated in HOX family genes, as HOTAIR, HOTTIP, HOXA11-AS, HOXB-AS4, and HOXC-AS3, were regulated by aberrant DNA methylation in GC [21]. However, the roles and mechanism of IncRNA HOXB-AS4 in GC remain unknown.

In the present study, we designed experiments to investigate the role of IncRNA HOXB-AS4 in the GC progression and the effect of epigenetic regulation on its abnormal expression (Additional fle 1: Fig.S1). Our results showed that $H O X B-A S 4$ is epigenetically activated by DNA methylation at the CpG islands within its promoter region. $H O X B-A S 4$ regulates the cell proliferation, migration, invasion, and EMT markers expression by competitively binding to miR-130a-5p, leading to the upregulation of plakophilin 4 (PKP4), which promotes the GC progression.

\section{Materials And Methods}

\section{Patients and specimens}

A total of 16 pairs of GC tissues and the pair-matched non-tumoral gastric tissues were acquired from the first people's hospital of suqian. The written consent agreement was signed and approved by the Ethics Committee of the Affiliated Suqian first People's Hospital of Nanjing Medical University. All patients involved in the present study didn't receive chemotherapy or radiotherapy before the surgery. 16 tissues were stored at $-80^{\circ} \mathrm{C}$ immediately after the surgery. 


\section{Cell culture}

The human gastric epithelial cell line GES-1, and human gastric cancer cell lines MKN-45, MKN-28 were purchased from the American Type Culture Collection (MD, USA). The human gastric cancer cell lines NClN87, AGS, HGC-27 were purchased from Chinese Academy of Sciences Cell Bank (Shanghai, China). All cells were cultured in RPMI-1640 culture medium supplemented with $10 \%$ fetal bovine serum (FBS, Invitrogen, USA) at $37^{\circ} \mathrm{C}$ with $5 \% \mathrm{CO}_{2}$.

\section{Plasmids construction and transfection}

The full-length IncRNA HOXB-AS4 was cloned into pcDNA3.1 (Invitrogen, USA), and the IncRNA HOXB-AS4 plasmid and corresponding empty vector were transfected into GES-1 or AGS cells using Lipofectamine 3000 reagent (Invitrogen, USA). The IncRNA HOXB-AS4 siRNAs, SP1 siRNAs, PKP4 siRNAs were synthesized by Genscript Biotech (Nanjing, China). miR-130-5p mimics, miR-130-5p inhibitors and relative control were purchased from Ribo Bio (Guangzhou, China). All siRNAs, miRNA mimics, inhibitors, or HOXB-AS4 plasmid were transiently transfected to cells by using Lipofectamine 3000 for $24 \mathrm{~h}$. The sequences of si-HOXB-AS4: AAGAGGACCACTCAGTTTAGG; si-SP1: AAGTGCAGCAGGATGGTTCTG; siPKP4: GAGACCACAGCCACCACTATT.

\section{RNA Extraction and quantitative Real-Time PCR (qRT-PCR)}

Total RNA was extracted using either TRIzol Reagent (Invitrogen, USA) following the manufacturer's instructions. Then mRNAs were reverse transcribed using the PrimeScript RT kit (Takara, Japan). The cDNAs were then analyzed by qRT-PCR using a QuantiTect SYBR Green PCR Kit (Qiagen, Germany), genespecific primers and a Rotor gene Q real-time PCR cycler (Qiagen, Germany). The reaction condition was as follows: $95^{\circ} \mathrm{C}$ for $30 \mathrm{~s}$, at $60{ }^{\circ} \mathrm{C}$ for $30 \mathrm{~s}, 45$ cycles at $60^{\circ} \mathrm{C}$ for $30 \mathrm{~s}$. RNA was quantified by $2-\Delta \Delta \mathrm{CT}$ method. After qRT-PCR reactions, the cycle threshold (CT) data were normalized to the internal control (U6 snRNA or GAPDH). Primers used were as follows: HOXB-AS4-F: CTT GTG CTT TAC AAG GCG GC, HOXBAS4-R: GAC CGG CCC CTA GGT TTA TC; miR-130a-5p-F: GCG CGG ATC CAG GCG GCA AAA GGA AGA GTG GTG, miR-130a-5p-R: CGG CGA ATT CCA CAA GCA CTG CAT ACA GAA GTA G; miR-140a-5p-F: CGG GAT CCG GTC CTC TGT TCG GTG GTG GCG; miR-140-5p-R: CGG AAT TCA ACC AGC AAG GGG ATG TCC CAA G; U6-F: GCT TCG GCA GCA CAT ATA CTA A, U6-R: AAC GCT TCA CGA ATT TGC GT; GAPDH-F: TGT GTC CGT CGT GGA TCT GA; GAPDH-R: CCT GCT TCA CCA CCT TCT TGA.

\section{Western blot}

Western blot assay was used to assess epithelial-mesenchymal transition (EMT)-related makers Vimentin, $\mathrm{N}$-Cadherin, and E-Cadherin and PKP4 protein expression. Total protein from tumor tissues or cells was extracted by using RIPA lysis buffer plus PMSF (Beyotime, China). BCA assay kit (Santa Cruz, USA) was used to detect total protein concentration. Prepared protein samples were separated by using SDS-PAGE electrophoresis, and transferred into PVDF membranes and incubated with prepared antibodies. Finally, enhanced chemiluminescence (ECL, ThermoFisher, USA) was used to visualize the 
membrane. The protein band analysis was conducted with ImageJ software. Antibodies against Vimentin, N-Cadherin, E-Cadherin, and GAPDH were purchased from CST (MA, USA). Antibody against PKP4 was purchased from Abcam (Cambridge, USA).

\section{Cell viability assay}

The viability of GSE-1 and AGS cells was assessed using MTT. Briefly, cells were plated in the 96-well microplates at a density of $1 \times 10^{4}$ cells per well. After transfection with indicated time, the cells were incubated with MTT $(0.5 \mathrm{mg} / \mathrm{ml})$ at $37^{\circ} \mathrm{C}$ for $3 \mathrm{~h}$. Then the medium was removed, and $100 \mathrm{mM}$ DMSO solution was added to dissolve the formazan crystals. The absorbance at $570 \mathrm{~nm}$ wavelength was detected using a microplate reader (Molecular Devices, Sunnyvale, USA).

\section{Clone formation assay}

The GSE- 1 or AGS cells were digested and plated in a $6 \mathrm{~cm}$ culture dish at a density of $5 \times 10^{3} /$ well after indicated treatments. The cells were subjected to the normal culture condition at $37^{\circ} \mathrm{C}, 5 \% \mathrm{CO}_{2}$ for 14 days. Then the cells in the dish were washed with PBS, and fixed with $3 \mathrm{~mL}$ methanol for 10 minutes. The cells were subjected to Giemsa dyeing for another 15 minutes. Then the cells were observed under a light microscope (Olympus, Japan), and the number of colonies was counted.

\section{Cell migration and invasion assay}

Cell migration and invasion were determined by transwell assay. GSE-1 and AGS cells were harvested and seeded to the upper transwell chamber at a density of $5 \times 10^{4}$ cells per well into an 8-mm pore transwell plate (Costar, USA) pre-treated with Matrigel (BD biosciences, USA) or not. Serum-free medium was added onto the upper chamber, and the culture medium containing $20 \%$ FBS was added into the lower chamber. Following $24 \mathrm{~h}$ incubation, the unmigrated or uninvaded cells were cleaned using a cotton swab and then fixed in $4 \%$ paraformaldehyde for $20 \mathrm{~min}$ and stained with hematoxylin. Images were taken, and the cell number was calculated.

\section{Methylation-specific PCR (MSP)}

Genomic DNA was extracted from the GC tissues and adjacent normal tissues by using the DNA extraction Kit (Qiagen, Germany) according to the manufacturer's instruction. The purified DNA was then exposed to bisulfite treatment by using the EpiTect Bisulfite Kit (Qiagen, Germany). Then the MSP of bisulfite-transformed DNA was performed with a nested, two-stage PCR method followed with the detection of the PCR products by using agarose gel electrophoresis.

\section{Chromatin immunoprecipitation (ChIP)}

ChIP assays were performed with an EZ-ChIP Kit (Millipore, USA) according to the manufacturer's instructions. Briefly, GES-1 and AGS cells were cross-linked with 1\% formaldehyde for $10 \mathrm{~min}$ followed with glycine treatment. Cell lysates were then sonicated to generate chromatin fragments and then 
immunoprecipitated with $S P 1$ antibody (CST, USA). IgG antibody (CST, USA) was used as the negative control. The DNA fragment was amplified by PCR. The HOXB-AS4 primer sequences are as follows: $\mathrm{F}$ : CTGAGTTTTCAGCCCTCCTG, R: AAGCTCCAATGAAGGGGTCT. The product was then analyzed by using agarose gel electrophoresis.

\section{Fluorescence in situ hybridization (FISH)}

To detect HOXB-AS4 expression, GSE-1 and AGS cells were fixed in $4 \%$ formaldehyde for $15 \mathrm{~min}$ at room temperature and then permeabilized with $70 \%$ ethanol. After rehydrated for $5 \mathrm{~min}$ at room temperature, the cells were incubated by using biotin-labeled HOXB-AS4 probe (Genepharma, Shanghai, China) at 37 ${ }^{\circ} \mathrm{C}$ for $8 \mathrm{~h}$. Then the Alexa Fluor 647-conjugated secondary antibody (Abcam, USA) was used to detect the biotin-labeled HOXB-AS4.

\section{Luciferase reporter assay}

The wild-type or mutant 3'UTR of PKP4 and the full length of HOXB-AS4 were amplified and cloned into pGL3-basic vector (Promega, WI, USA) separately. The binding site of 3'UTR of PKP4 was mutated from UAU UAU GUU UUU UAA AAU GUG AG to TTT ATA GTA TTA AAT TTT GAG TG for miR-130a-5p, and the potential binding sites of HOXB-AS4 was mutated from AAAAGAGA to TTTTGTG for miR-130a-5p. Then, AGS cells were plated on a 24-well plate and co-transfected with wild-type or mutant luciferase plasmids and miR-23c mimic, miR-23c inhibitor or control miRNA. A Dual-Luciferase Reporter Assay System (Promega, WI, USA) was used to measure the luciferase activity. The relative luciferase activity of each sample was normalized to Renilla luciferase activity.

\section{Tumor xenograft model}

$1 \times 10^{7}$ AGS cells were suspended in $200 \mu \mathrm{l}$ PBS and subcutaneously injected into right flank of 4-6week-old BALB/c nu/nu male mice (Charles River Lab, Beijing, China). The mice were recorded the tumor volume (volume $=\left(\right.$ length $\times$ width $\left.^{2}\right) / 2$ ) every 3 days. Knockdown of miR-130a-5p was performed by using miR-130a-5p antagomir (Ribo Bio, Guangzhou, China) which was administrated by tail injection at the dosage of $80 \mathrm{mg} / \mathrm{kg}$. Knockdown of HOXB-AS4 or PKP4 was performed by using the related knockdown lentivirus (GenePharma, Shanghai, China) by intra tumoral injection of $50 \mu \mathrm{L}$ virus $\left(4 \times 10^{7} \mathrm{IU} / \mathrm{mL}\right)$ after the tumor cells injection. After 15 days, the mice were sacrificed. The tumor tissues were subjected to western blot analysis, hematoxylin-eosin (H\&E) staining and immunohistochemical (IHC) analysis. The animal experiments were approved by the Animal Care and Use Committee of the first people's hospital of suqian.

\section{H\&E and IHC analysis}

For H\&E staining, the prepared tumor tissue slices were dewaxed and hydrated. After washed by water, tumor tissue slices were stained in hematoxylin solution for $5 \mathrm{~min}$. Next, after treated by $1 \%$ hydrochloric alcohol for $15 \mathrm{~s}$, the slices were washed with water. And then the slices were stained by eosin solution for 
1 min. Finally, tissue slices were dehydrated, transparentized and sealed by neutral gum, observed under an optical microscope (Olympus, Tokyo, Japan). For IHC staining, the paraffin-embedded tumor sections were dewaxed and treated with $3 \% \mathrm{H}_{2} \mathrm{O}_{2}$ to deactivate endogenous peroxidase. After blocking nonspecific antigen binding with $5 \% \mathrm{BSA}$ at $37^{\circ} \mathrm{C}$ for $1 \mathrm{~h}$, the sections were incubated with a specific primary antibody against PKP4 (1:100 dilution, Abcam, USA) at $4{ }^{\circ} \mathrm{C}$ overnight. After incubating with the corresponding secondary antibodies at $37^{\circ} \mathrm{C}$ for $1 \mathrm{~h}$, the sections were stained with diaminobenzidine and counterstained with hematoxylin. Representative images were taken using a light microscope (Olympus, Tokyo, Japan).

\section{Kaplan-meier analysis}

The overall survival data were derived from the online database Genomic Data Commons Data Portal (https://portal.gdc.cancer.gov/). Of the $110 \mathrm{GC}$ samples, 108 samples showed high HOXB-AS4 expression, whereas 82 samples showed low HOXB-AS4 expression. The survival rates were analyzed by Kaplan-Meier curves. Then, the log-rank test was performed to evaluate the mean of the two groups.

\section{Bioinformatics}

The online database Starbase (http://starbase.sysu.edu.cn/) was used to predict possible miRNAs that may be sponged by HOXB-AS4. In addition, the online database miRCancer (http://mircancer.ecu.edu/) was used to predict the abnormal expression of miRNAs in gastric cancer. Then, the miRNAs that may be sponged by HOXB-AS4 in gastric cancer were screened through the intersection of the Starbase and miRCancer. The online databases miRDB (http://mirdb.org/) and Targetscan (http://www.targetscan.org/『were used to screen the potential targets of miR-130a-5p.

\section{Statistical analysis}

All data were shown as mean \pm s.e.m. and experiments were carried out at least three times. GraphPad 6.0 version was adopted for statistical analyses. Student's t-test was performed to evaluate the mean of the two groups and one-way ANOVA followed Tukey's poc host was used to analyze significant differences among multiple groups. $\mathrm{P}<0.05$ was regarded as statistically significant.

\section{Results}

\section{LncRNA HOXB-AS4 is up-regulated in GC tissues and GC cells}

To determine whether IncRNA HOXB-AS4 plays a role in the pathogenesis of GC, we first investigated the expression of IncRNA HOXB-AS4 in the tumor tissues. According to the GEPIA database (http://gepia.cancer-pku.cn/), we found that IncRNA HOXB-AS4 was significantly up-regulated in various types of tumors, especially in GC (Fig. 1A). The HOXB-AS4 expression in the GC tissues was further verified by using qRT-PCR. The results showed that HOXB-AS4 was significantly up-regulated in the 16 pairs of GC tissues compared to the pair-matched non-tumoral gastric tissues (Fig. 1B). Moreover, 
Kaplan-meier analysis showed that HOXB-AS4 expression was associated with the prognosis of 110 of GC patients (Fig. 1C). 108 of GC patients with higher HOXB-AS4 expression showed a poorer prognosis than 82 of GC patients with lower HOXB-AS4 expression (Fig. 1C), indicating that HOXB-AS4 expression may contribute to the GC progression. We further investigated the expression level of HOXB-AS4 in various GC cells and in normal human gastric epithelial cell line GES-1. As shown in Fig. 1D, HOXB-AS4 exhibited higher expression level in the cancer cells compared to the normal gastric cells, among which AGS cells showed the highest expression.

\section{HOXB-AS4 modulates GC cells proliferation, migration, invasion and EMT markers expression}

To investigate the effect of HOXB-AS4 on tumor progression, we knocked down HOXB-AS4 in AGS cells and overexpressed $H O X B-A S 4$ in GES-1 cells. The transfection efficacy was verified by using qRT-PCR (Fig. 2A). MTT assay showed that overexpression of HOXB-AS4 increased the GES-1 cell viability, while knockdown of HOXB-AS4 reduced the AGS cell viability (Fig. 2B). Overexpression of HOXB-AS4 promoted the GES-1 cell clone formation, while knockdown of HOXB-AS4 inhibited the AGS cell clone formation (Fig. 2C). GES-1 cells showed enhanced migration and invasion ability after overexpressed with HOXBAS4 (Fig. 2D). Knockdown of HOXB-AS4 in AGS cells restrained the cell migration and invasion ability (Fig. 2D).

As activation of EMT process provides tumor cells with invasive and migratory ability[22], we then investigated the effect of HOXB-AS4 on EMT markers expression. In GES-1 cells, overexpression of HOXBAS4 reduced Vimentin expression and increased the $\mathrm{N}$-Cadherin and $\mathrm{E}$-Cadherin expression (Fig. 2E). In AGS cells, knockdown of HOXB-AS4 increased the Vimentin expression and inhibited the N-Cadherin and E-Cadherin expression (Fig. 2E).

\section{HOXB-AS4 acts as a sponge for miR-130a-5p}

We then explored the mechanism of the regulatory effect of HOXB-AS4. We examined the intracellular localization of HOXB-AS4. Results of FISH showed that HOXB-AS4 localized in the cytoplasm of the GSE1 and AGS cells (Fig. 3A). It is reported that cytoplasmic IncRNA exerts its regulatory function through several mechanisms, including mRNA turnover, translation, protein stability, sponging of cytosolic factors, and modulation of signaling pathways[23]. It's worth noting that ceRNA mechanism is one of the major functions of cytoplasmic IncRNA. Therefore, we then hypothesized that HOXB-AS4 may play its role through the ceRNA mechanism. By using the Starbase and miRCancer database, we identified two miRNAs that may be sponged by HOXB-AS4 (Fig. 3B). qRT-PCR showed that overexpression of HOXB-AS4 in AGS led to lower miR-130a-5p level in cells, and miR-130a-5p showed an increased level in HOXB-AS4knockdown cells (Fig. $3 \mathrm{C}$ ). We then predicted the potential binding sites between HOXB-AS4 and miR130a-5p (Fig. 3D). Luciferase reporter showed that miR-130a-5p significantly decreased the luciferase activity of the cells transfected with WT rather than the mutant HOXB-AS4, indicating that miR-130a-5p specifically bound to the HOXB-AS4 (Fig. 3E). Moreover, miR-130a-5p showed significant downregulation in 16 pairs of GC tissues compared to the pair-matched non-tumoral gastric tissues (Fig. 3F), which negatively correlated with the HOXB-AS4 expression (Fig. 3G). 
To determine the targets of miR-130a-5p which might contribute to the progression of GC, we screened the potential targets of miR-130a-5p by using TargetScan and miRDB, which identified the PKP4 may be the potential targets of miR-130a-5p (Fig. 4A). We then predicted the potential binding sites (Fig. 4B), and performed luciferase reporter assays to verify whether PKP4 was a target of miR-130a-5p. miR-130a-5p significantly decreased the luciferase activity of the cells transfected with WT rather than the mutant PKP4, indicating that miR-130a-5p specifically bound to the 3'UTR of PKP4 (Fig. 4C). Furthermore, western blot showed that miR-130a-5p overexpression markedly reduced the PKP4 protein expression, and miR-130a-5p knockdown increased the PKP4 expression (Fig. 4D). Then, 16 pairs of GC tissues and the pair-matched non-tumoral gastric tissues were collected for western blot assay. PKP4 showed an obvious upregulation in the $\mathrm{GC}$ tissues compared to the pair-matched non-tumoral gastric tissues (Fig. 4E). PKP4 showed significant positive correlation with HOXB-AS4 expression, and negative correlation with miR-130a-5p expression (Fig. 4F). All these data suggested that HOXB-AS4/miR-130a$5 \mathrm{p} / P K P 4$ may be involved in the GC progression.

HOXB-AS4 modulates GC cells proliferation, migration, invasion and EMT markers expression via miR130a/PKP4 axis.

We then explored the effect of HOXB-AS4/miR-130a-5p/PKP4 axis in GC cells. As shown in Fig. 5A, HOXB-AS4 knockdown decreased the cell viability. MiR-130a-5p knockdown eliminated the effect of HOXB-AS4 knockdown, while PKP4 knockdown further abolished the effect of miR-130a-5p inhibitor (Fig. 5A). HOXB-AS4 knockdown inhibited the cell clone formation, which was abolished by miR-130a-5p knockdown, and PKP4 knockdown further inhibited the effect of miR-130a-5p inhibitor (Fig. 5B). AGS cells knocked down of $H O X B-A S 4$ showed a reduced migration and invasion ability, which were abrogated by miR-130a-5p knockdown, and PKP4 knockdown further reduced the cell migration and invasion ability, which were restored by miR-130a-5p inhibitor (Fig. 5C). In AGS cells, knockdown of HOXB-AS4 increased E-Cadherin expression and decreased N-Cadherin and Vimentin expression (Fig. 5D). Knockdown of miR130a-5p inhibited the effect of HOXB-AS4 knockdown, and knockdown of PKP4 knockdown further repressed the effect of miR-130a-5p. All these results indicated that inhibition of HOXB-AS4/miR-130a$5 \mathrm{p} /$ PKP4 axis suppressed the proliferation and modulated EMT markers in GC cells.

\section{Knockdown of HOXB-AS4 inhibits GC progression and modulates EMT markers in vivo}

We further verified the effect and underlying mechanism of HOXB-AS4 in vivo. Mice were divided into four groups, with 10 mice in each group. Mice in HOXB-AS4 knockdown group showed an obvious smaller tumor size compared to the control group, while treatment of miR-130a-5p antagomir inhibited the tumorsuppressive effect of HOXB-AS4 knockdown (Fig. 6A, B). Knockdown of PKP4 further inhibited the tumor growth promoted by miR-130a-5p knockdown, showing smaller tumor size compared to the knockdown of HOXB-AS4 and miR-130-5p group (Fig. 6A, B). Western blot showed that knockdown of HOXB-AS4 increased E-Cadherin expression and decreased N-Cadherin and Vimentin expression (Fig. 6C). Knockdown of miR-130a-5p inhibited the effect of HOXB-AS4 knockdown on, and knockdown of PKP4 
knockdown further repressed the effect of miR-130a-5p (Fig. 6C). IHC staining revealed that the PKP4 expression showed the consistency with the tumor progression (Fig. 6D). HOXB-AS4 knockdown induced lower PKP4 expression in the tumors, while knockdown of miR-130-5p recovered the PKP4 expression inhibited by HOXB-AS4 knockdown (Fig. 6D). All these data suggested that inhibition of $H O X B-A S 4 / \mathrm{miR}-$ $130 \mathrm{a}-5 \mathrm{p} /$ PKP4 axis restrained the tumor progression in vivo.

\section{DNA methylation and SP1 participate in the regulation of the expression of HOXB-AS4}

We then explored the mechanism underlying the high expression of HOXB-AS4 in the GC tissues and cells. By using the UCSC Genome Bioinformatics Site(http://genome.ucsc.edu/), we identified CpG islands together with high enrichment and overlapping H3K427AC peaks within the promoter region of HOXB-AS4 (Fig. 7A), indicating a potential relationship between DNA methylation and HOXB-AS4 expression. We then performed MSP analysis to verify whether DNA methylation was involved in the upregulation of HOXB-AS4 in GC. As shown in Fig. 7B, DNA methylation levels were significantly decreased in the promoter region of HOXB-AS4 in the GC tissues. Accordingly, in the AGS cells, DNA methylation levels in the promoter region of HOXB-AS4 were lower than that in the GES-1 cells (Fig. 7C). We then treated the GES-1 cells or AGS cells with the DNA demethylation agent azacytidine for different time. We found that HOXB-AS4 expression increased with time in GSE-1 and AGS cells, indicating that the inhibition of DNA methylation promoted the HOXB-AS4 expression (Fig. 7D). We further used PROMO (http://alggen.Isi.upc.es/cgi-bin/promo_v3) to predict the potential transcription factors of HOXB-AS4, which showed that $S P 1$ may be a transcription factor of HOXB-AS4. Results of CHIP revealed the enrichment of $S P 1$ at the promoter of HOXB-AS4 (Fig. 7E). We then changed the SP1 expression in GES-1 and AGS cells, and the overexpression or knockdown efficiency was verified by western blot (Fig. 7G). Overexpression of SP1 enhanced HOXB-AS4 expression in GES-1 and AGS cells, and SP1 knockdown restrained $H O X B-A S 4$ expression (Fig. 7F). All these data suggested that DNA methylation and transcription factor $S P 1$ were involved in the regulation of $S P 1$ expression.

\section{Discussion}

Increasing evidence showed that IncRNAs play important roles in the development of GC[24, 25]. In the study, we revealed a novel function of $H O X B-A S 4$ in GC progression. We reported that DNA-methylationmediated HOXB-AS4 promoted GC proliferation, migration, and invasion, and modulated EMT markers expression through regulating miR-130a-5p/PKP4 (Fig. 8).

HOXB-AS4 was HOXB cluster antisense RNA 4, whose effect has barely been reported. In the present study, by using the data of GEPIA database, we found that HOXB-AS4 was upregulated in various cancer, including GC. We further verified HOXB-AS4 overexpression in GC tissues and cells, and found its upregulation correlated with the poor prognosis of GC patients, indicating it may play potential roles in GC progression. Further in-vitro experiment showed that HOXB-AS4 overexpression promoted the AGS and GSE-1 cells proliferation, migration and invasion. 
EMT encompasses complex and dynamic changes in phenotypes of cellular organization from epithelial characteristics to mesenchymal traits[26]. The epithelial and mesenchymal cell characteristics include EMT-inducing signals, EMT-Transcription factors and EMT markers[26]. One of the specific changes is the decrease of epithelial marker proteins, such as E-cadherin, and the up-regulation of mesenchymal marker proteins, such as N-cadherin and Vimentin[26]. It is reported that activation of EMT process confers tumor cells with invasive and migratory ability, and supports tumor metastasis[27]. Various studies have reported that IncRNA regulates the EMT process in cancer development[28, 29]. For example, IncRNA SNHG16 promotes colorectal cancer cell proliferation, migration, and modulates EMT markers expression through miR-124-3p/MCP-7[30]. LncRNA LINC00525 regulates the proliferation and epithelial to mesenchymal transition of human glioma cells by sponging miR-338-3p [31]. In the present study, overexpression of HOXB-AS4 increased GSE-1 cell proliferation, migration and invasion, and modulated EMT markers expression. In AGS cells, knockdown of HOXB-AS4 inhibited the cancer cell proliferation, migration and invasion, accompanied with the modulation of EMT markers expression, indicating that $H O X B-A S 4$ may function tumor-promoting effect through modulating EMT process.

LncRNA could act as miRNAs sponge to regulate gene expression, which were defined as "ceRNA" mechanism [32, 33]. CeRNA mechanism is the main regulatory model of cytoplasm IncRNA[34, 35]. In the present study, we identified the HOXB-AS4 as a cytoplasm IncRNA by using FISH, indicating its regulatory role in GC may rely on the ceRNA mechanism. Screen of the Starbase and miRCancer database showed that miR-130a-5p and miR-140a-5p may interactwith HOXB-AS4. Luciferase reporter assay further showed that HOXB-AS4 was a miR-130a-5p sponge. Further experiments showed that miR-130a-5p targeted the 3'UTR of PKP4. MiR-130a-5p was reported to inhibit tumor invasion and metastatic potential in non-small cell lung cancer[36]. MiR-130a-5p enhanced the sensitivity of cisplatin-resistant gastric cancer cells to chemotherapy [37]. PKP4 was also called "p0071", which were reported to interact with Ecadherin to promote the invasion and metastasis of cancer cells[38]. In the present in-vitro and in-vivo experiments, we verified that knockdown of miR-130a-5p inhibited the anti-tumor effect of HOXB-AS4 knockdown, showing an increase in cell proliferation and modulation of EMT markers expression. Knockdown of PKP4 further abolished the effect of miR-130a-5p knockdown on recovering the cell proliferation and EMT markers expression. All these results indicated that HOXB-AS4/miR-130a-5p/PKP4 axis promoted GC development.

We further investigate the mechanism of HOXB-AS4 upregulation in GC. LncRNA expression is regulated by multiple factors, including chromatin state, the utilization of transcription factors, and some posttranscriptional regulation [39]. DNA methylation is a stable and heritable epigenetic mark which could influence the chromatin state to change the transcriptional state of genes [40]. Many IncRNAs have been reported to be regulated by DNA methylation [41]. Although DNA methylation was studied much as a epigenetic modifications in GC, the DNA methylation-mediated dysregulation of IncRNAs was barely reported[42-44]. In the present study, we identified abnormally low methylation levels of the promoter region of HOXB-AS4, which resulted in enhanced IncRNA expression. SP1 is a transcription factor which has been reported to mediate various IncRNA aberrant expression [45, 46]. SP1-dependent DNA methylation was an important pattern for gene overexpression[47]. Consistently, we found that SP1 was 
involved in the DNA-methylation-mediated upregulation of $H O X B-A S 4$, indicating that SP1-dependent DNA methylation was the molecular mechanism of HOXB-AS4 aberrant upregulation in GC. To data, there are some limitations in the study. For instance, the number of tissue samples seems inadequate. Only 16 pairs of GC tissues and the pair-matched non tumoral gastric tissues were acquired to detect HOXB-AS4, miR-130a-5p, and PKP4 expression. Furthermore, the involvement of EMT in any process cannot rely solely on a few salient molecular markers, such as E-cadherin and vimentin. As is known, cytoplasmic IncRNA exerts its regulatory function through several mechanisms, including mRNA turnover, translation, protein stability, sponging of cytosolic factors, and modulation of signaling pathways[23]. In the current study, we could not excluded the other mode of action, and just clarified the role of HOXB-AS4 /miR-130a$5 \mathrm{p} /$ PKP4 axis on GC progression. We will further explore the mechanism of HOXB-AS4 in the future study.

In conclusion, we identified that abnormal upregulation of IncRNA HOXB-AS4 contributed to GC progression. HOXB-AS4 was found to be epigenetically activated by DNA methylation at the CpG islands within its promoter region. HOXB-AS4 regulated the cell proliferation, migration, invasion, and EMT markers expression by competitively binding to miR-130a-5p, leading to the upregulation of PKP4 in GC progression. Our study provides new insights for the understanding of epigenetic regulation on IncRNA expression in GC, and indicates that HOXB-AS4 could be a biomarker of GC prognosis.

\section{Abbreviation}

GC, Gastric cancer; IncRNAs, Long noncoding RNAs; qRT-PCR, quantitative Real-Time PCR; EMT, epithelialmesenchymal transition; FISH, fluorescence in situ hybridization; MSP, methylation-specific PCR; CeRNA, competitive endogenous RNA; miRNA, microRNA; PKP4, plakophilin 4; FBS, fetal bovine serum; CT, cycle threshold; ChIP, Chromatin immunoprecipitation; H\&E, hematoxylin-eosin; IHC; immunohistochemical.

\section{Declarations}

\section{Acknowledgments}

Not applicable

\section{Authors' contributions}

Yu Fan and Rong He conceived and directed the study. Yu Fan, Xiao-yan Wang and Rong He contributed to the project design.Xiaoyan Wang, Rong He,Yan Wang, Yunyun Liu, Yuxin Wang and Dandan Chen performed the experiments. Rong He analyzed the bioinformatics data.Rong He and Xiao-yan Wang analyzed and interpreted the data.. All of them read and approved the final manuscript.

\section{Funding}


Suqian Science and Technology Support Project Fund (K201907), Jiangsu Innovative Team Leading Talent Fund (CXTDC2016006, QNRC2016446), Jiangsu 333 Talent Fund (BRA2020016),Jiangsu Provincial Key Research and Development Special Fund (BE2015666), Jiangsu Six High Peak Talent Fund (WSW-205,WSW236), Zhenjiang Key Research and Development Fund (SH2021038).

\section{Availability of data and materials}

The data used to support the findings of this study are available from the corresponding author upon request.

\section{Ethics approval and consent to participate}

The written consent agreement was signed and approved by the Ethics Committee of The Affiliated Suqian first People's Hospital of Nanjing Medical University.

\section{Consent for publication}

The authors declared that there are no conflicts of interest.

\section{Consent for publication}

All the listed authors have participated in the study, and have seen and approved the submitted manuscript.

\section{Competing interests}

The authors declare that they have no conflict of interest.

\section{References}

1. Bray F, Ferlay J, Soerjomataram I, Siegel RL, Torre LA, Jemal A. Global cancer statistics 2018: GLOBOCAN estimates of incidence and mortality worldwide for 36 cancers in 185 countries. Cancer J Clin. 2018;68(6):394-424. doi:10.3322/caac.21492.

2. Smyth EC, Nilsson M, Grabsch HI, van Grieken NC, Lordick F. Gastric cancer. Lancet. 2020;396(10251):635-48. doi:10.1016/s0140-6736(20)31288-5.

3. Song Z, Wu Y, Yang J, Yang D, Fang X. Progress in the treatment of advanced gastric cancer. Tumour biology: the journal of the International Society for Oncodevelopmental Biology Medicine. 2017;39(7):1010428317714626. doi:10.1177/1010428317714626.

4. Takahari D. [Chemotherapy for patients with advanced gastric cancer]. Nihon Shokakibyo Gakkai zasshi $=$ The Japanese journal of gastro-enterology. 2018;115(6):514-20. doi: 10.11405/nisshoshi.115.514.

5. Berger H, Marques MS, Zietlow R, Meyer TF, Machado JC, Figueiredo C. Gastric cancer pathogenesis. Helicobacter. 2016;21(Suppl 1):34-8. doi:10.1111/hel.12338. 
6. Schmitz SU, Grote P, Herrmann BG. Mechanisms of long noncoding RNA function in development and disease. Cell Mol Life Sci. 2016;73(13):2491-509. doi:10.1007/s00018-016-2174-5.

7. Akhade VS, Pal D, Kanduri C. Long Noncoding RNA: Genome Organization and Mechanism of Action. Advances in experimental medicine and biology. 2017;1008:47-74. doi: 10.1007/978-981-10-52033_2.

8. Chen Z, Bu N, Qiao X, Zuo Z, Shu Y, Liu Z, et al. Forkhead Box M1 Transcriptionally Regulates the Expression of Long Noncoding RNAs Snhg8 and Gm26917 to Promote Proliferation and Survival of Muscle Satellite Cells. Stem cells (Dayton, Ohio). 2018;36(7):1097-108. doi: 10.1002/stem.2824.

9. Qian X, Zhao J, Yeung PY, Zhang QC, Kwok CK. Revealing IncRNA Structures and Interactions by Sequencing-Based Approaches. Trends Biochem Sci. 2019;44(1):33-52. doi:10.1016/j.tibs.2018.09.012.

10. Zheng $X$, Wang $X$, Zheng L, Zhao H, Li W, Wang B, et al. Construction and Analysis of the TumorSpecific mRNA-miRNA-IncRNA Network in Gastric Cancer. Front Pharmacol. 2020;11:1112. doi:10.3389/fphar.2020.01112.

11. Ren N, Jiang T, Wang C, Xie S, Xing Y, Piao D, et al. LncRNA ADAMTS9-AS2 inhibits gastric cancer (GC) development and sensitizes chemoresistant GC cells to cisplatin by regulating miR-2233p/NLRP3 axis. Aging. 2020;12(11):11025-41. doi:10.18632/aging.103314.

12. Shi $X$, You X, Zeng WC, Deng YJ, Hong HL, Huang OX, et al. LncRNA PAPAS aggravates the progression of gastric cancer through regulating miRNA-188-5p. Eur Rev Med Pharmacol Sci. 2021;25(6):2462. doi:10.26355/eurrev_202103_25402.

13. Yu M, Zhan J, Zhang H. HOX family transcription factors: Related signaling pathways and posttranslational modifications in cancer. Cellular signalling. 2020;66:109469. doi:10.1016/j.cellsig.2019.109469.

14. Bhatlekar S, Fields JZ, Boman BM. HOX genes and their role in the development of human cancers. J Mol Med. 2014;92(8):811-23. doi:10.1007/s00109-014-1181-y.

15. Zhu H, Dai W, Li J, Xiang L, Wu X, Tang W, et al. HOXD9 promotes the growth, invasion and metastasis of gastric cancer cells by transcriptional activation of RUFY3. Journal of experimental clinical cancer research: CR. 2019;38(1):412. doi:10.1186/s13046-019-1399-1.

16. Wang Y, Dang Y, Liu J, Ouyang X. The function of homeobox genes and IncRNAs in cancer. Oncology letters. 2016;12(3):1635-41. doi:10.3892/ol.2016.4901.

17. Zhao R, Zhang X, Zhang Y, Zhang Y, Yang Y, Sun Y, et al. HOTTIP Predicts Poor Survival in Gastric Cancer Patients and Contributes to Cisplatin Resistance by Sponging miR-216a-5p. Frontiers in cell developmental biology. 2020;8:348. doi:10.3389/fcell.2020.00348.

18. Sun M, Nie F, Wang Y, Zhang Z, Hou J, He D, et al. LncRNA HOXA11-AS Promotes Proliferation and Invasion of Gastric Cancer by Scaffolding the Chromatin Modification Factors PRC2, LSD1, and DNMT1. Cancer research. 2016;76(21):6299-310. doi:10.1158/0008-5472.Can-16-0356.

19. Liu Z, Chen Z, Fan R, Jiang B, Chen X, Chen Q, et al. Over-expressed long noncoding RNA HOXA11-AS promotes cell cycle progression and metastasis in gastric cancer. Mol Cancer. 2017;16(1):82. 
doi:10.1186/s12943-017-0651-6.

20. Tsai KW, Tsai CY, Chou NH, Wang KC, Kang CH, Li SC, et al. Aberrant DNA Hypermethylation Silenced LncRNA Expression in Gastric Cancer. Anticancer research. 2019;39(10):5381-91. doi:10.21873/anticanres.13732.

21. Song P, Wu L, Guan W. Genome-Wide Identification and Characterization of DNA Methylation and Long Non-Coding RNA Expression in Gastric Cancer. Frontiers in genetics. 2020;11:91. doi:10.3389/fgene.2020.00091.

22. Pastushenko I, Blanpain C. EMT Transition States during Tumor Progression and Metastasis. Trends in cell biology. 2019;29(3):212-26. doi:10.1016/j.tcb.2018.12.001.

23. Long $Y$, Wang $X$, Youmans DT, Cech TR. How do IncRNAs regulate transcription? 2017;3(9):eaao2110. doi: 10.1126/sciadv.aao2110.

24. Zhang X, Zhang W, Jiang Y, Liu K, Ran L, Song F. Identification of functional IncRNAs in gastric cancer by integrative analysis of GEO and TCGA data. Journal of cellular biochemistry. 2019;120(10):17898-911. doi:10.1002/jcb.29058.

25. Fattahi S, Kosari-Monfared M, Golpour M, Emami Z, Ghasemiyan M, Nouri M, et al. LncRNAs as potential diagnostic and prognostic biomarkers in gastric cancer: A novel approach to personalized medicine. Journal of cellular physiology. 2020;235(4):3189-206. doi:10.1002/jcp.29260.

26. Yang J, Antin P, Berx G, Blanpain C, Brabletz T, Bronner M, et al. Guidelines and definitions for research on epithelial-mesenchymal transition. Nature reviews Molecular cell biology. 2020;21(6):341-52. doi:10.1038/s41580-020-0237-9.

27. Diepenbruck M, Christofori G. Epithelial-mesenchymal transition (EMT) and metastasis: yes, no, maybe? Current opinion in cell biology. 2016;43:7-13. doi: 10.1016/j.ceb.2016.06.002.

28. Gong J, Wang Y, Shu C. LncRNA CHRF promotes cell invasion and migration via EMT in gastric cancer. Eur Rev Med Pharmacol Sci. 2020;24(3):1168-76. doi:10.26355/eurrev_202002_20168.

29. McCabe EM, Rasmussen TP. IncRNA involvement in cancer stem cell function and epithelialmesenchymal transitions. Sem Cancer Biol. 2021;75:38-48. doi:10.1016/j.semcancer.2020.12.012.

30. Chen ZY, Wang XY, Yang YM, Wu MH, Yang L, Jiang DT, et al. LncRNA SNHG16 promotes colorectal cancer cell proliferation, migration, and epithelial-mesenchymal transition through miR-124-3p/MCP1. Gene therapy. 2020. doi:10.1038/s41434-020-0176-2.

31. Wan Y, Liang F, Wei M, Liu Y. Long non-coding RNA LINC00525 regulates the proliferation and epithelial to mesenchymal transition of human glioma cells by sponging miR-338-3p. AMB Express. 2020;10(1):156. doi:10.1186/s13568-020-01094-4.

32. Wu XS, Wang F, Li HF, Hu YP, Jiang L, Zhang F, et al. LncRNA-PAGBC acts as a microRNA sponge and promotes gallbladder tumorigenesis. EMBO Rep. 2017;18(10):1837-53. doi:10.15252/embr.201744147.

33. Cao Y, Pan L, Zhang X, Guo W, Huang D. LncRNA SNHG3 promotes autophagy-induced neuronal cell apoptosis by acting as a ceRNA for miR-485 to up-regulate ATG7 expression. Metabolic brain disease. 2020;35(8):1361-9. doi:10.1007/s11011-020-00607-1. 
34. Rashid F, Shah A, Shan G. Long Non-coding RNAs in the Cytoplasm. Genom Proteom Bioinform. 2016;14(2):73-80. doi:10.1016/j.gpb.2016.03.005.

35. Carlevaro-Fita J, Johnson R. Global Positioning System: Understanding Long Noncoding RNAs through Subcellular Localization. Molecular cell. 2019;73(5):869-83. doi:10.1016/j.molcel.2019.02.008.

36. Ma F, Xie Y, Lei Y, Kuang Z, Liu X. The microRNA-130a-5p/RUNX2/STK32A network modulates tumor invasive and metastatic potential in non-small cell lung cancer. 2020;20(1):580. doi: 10.1186/s12885-020-07056-0.

37. Fang QL, Li KC, Wang L, Gu XL, Song RJ, Lu S. Targeted Inhibition of CCL22 by miR-130a-5p Can Enhance the Sensitivity of Cisplatin-Resistant Gastric Cancer Cells to Chemotherapy. Cancer management research. 2020;12:3865-75. doi:10.2147/cmar.s249738.

38. Zhao H, Zhang D, Yang L, Wang E. p0071 interacts with E-cadherin in the cytoplasm so as to promote the invasion and metastasis of non-small cell lung cancer. Molecular carcinogenesis. 2018;57(1):89-96. doi:10.1002/mc.22734.

39. Peng WX, Koirala P, Mo YY. LncRNA-mediated regulation of cell signaling in cancer. Oncogene. 2017;36(41):5661-7. doi:10.1038/onc.2017.184.

40. Glastad KM, Goodisman MA, Yi SV, Hunt BG.. (Bethesda. Effects of DNA Methylation and Chromatin State on Rates of Molecular Evolution in Insects. Md). 2015;G3(2):357-63. doi:10.1534/g3.115.023499. 6 ) .

41. Wei JW, Huang K, Yang C, Kang CS. Non-coding RNAs as regulators in epigenetics (Review). Oncol Rep. 2017;37(1):3-9. doi:10.3892/or.2016.5236.

42. Zong L, Hattori N, Yoda Y, Yamashita S, Takeshima H, Takahashi T, et al. Establishment of a DNA methylation marker to evaluate cancer cell fraction in gastric cancer. Gastric cancer: official journal of the International Gastric Cancer Association the Japanese Gastric Cancer Association. 2016;19(2):361-9. doi:10.1007/s10120-015-0475-2.

43. Sugimoto $\mathrm{K}$, Ito $\mathrm{T}$, Hulbert $\mathrm{A}$, Chen $\mathrm{C}$, Orita $\mathrm{H}$, Maeda $\mathrm{M}$, et al. DNA methylation genome-wide analysis in remnant and primary gastric cancers. Gastric cancer: official journal of the International Gastric Cancer Association the Japanese Gastric Cancer Association. 2019;22(6):1109-20. doi:10.1007/s10120-019-00949-5.

44. Ebrahimi V, Soleimanian A, Ebrahimi T, Azargun R, Yazdani P, Eyvazi S, et al. Epigenetic modifications in gastric cancer: Focus on DNA methylation. Gene. 2020;742:144577. doi:10.1016/j.gene.2020.144577.

45. Chang S, Sun L, Feng G. SP1-mediated long noncoding RNA POU3F3 accelerates the cervical cancer through miR-127-5p/FOXD1. Biomedicine \& pharmacotherapy =. Biomedecine pharmacotherapie. 2019;117:109133. doi:10.1016/j.biopha.2019.109133.

46. Tian $Y$, Ma R, Sun Y, Liu H, Zhang H, Sun Y, et al. Correction: SP1-activated long noncoding RNA IncRNA GCMA functions as a competing endogenous RNA to promote tumor metastasis by 
sponging miR-124 and miR-34a in gastric cancer. Oncogene. 2020;39(42):6621.

doi:10.1038/s41388-020-1377-2.

47. Paonessa F, Latifi S, Scarongella H, Cesca F, Benfenati F. Specificity protein 1 (Sp1)-dependent activation of the synapsin I gene (SYN1) is modulated by RE1-silencing transcription factor (REST) and 5'-cytosine-phosphoguanine (CpG) methylation. J Biol Chem. 2013;288(5):3227-39. doi:10.1074/jbc.M112.399782.

\section{Figures}

A

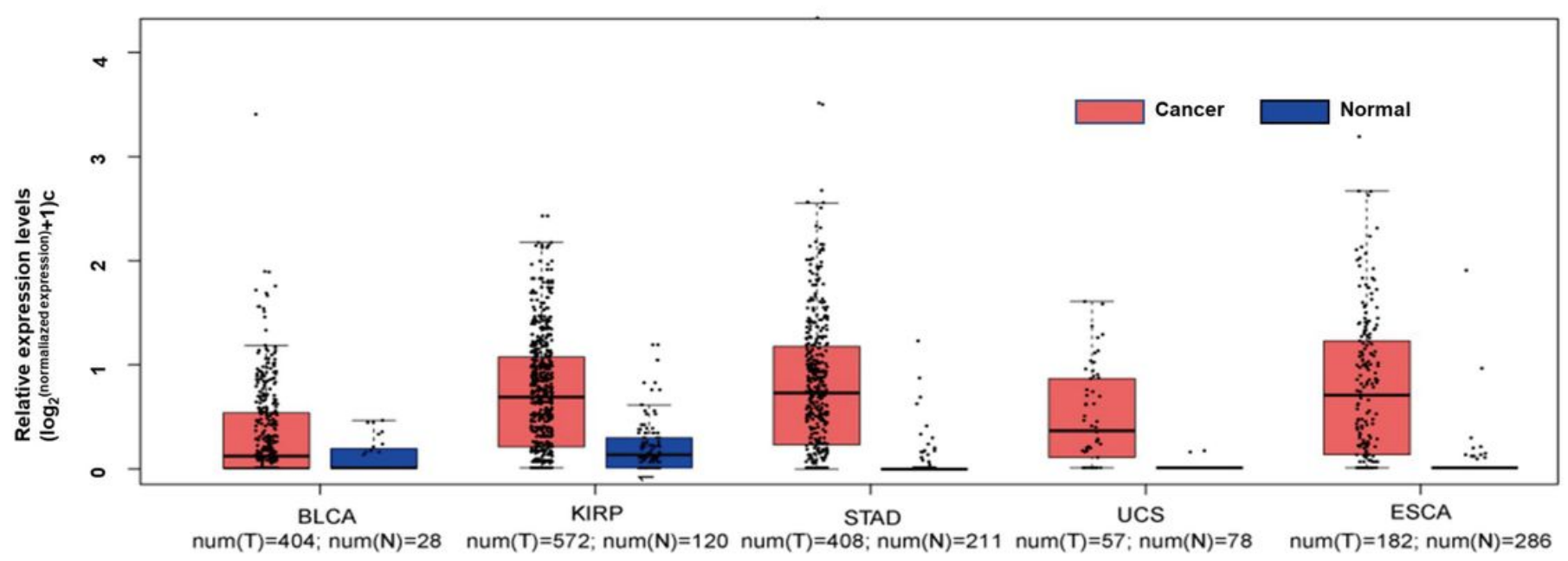

B

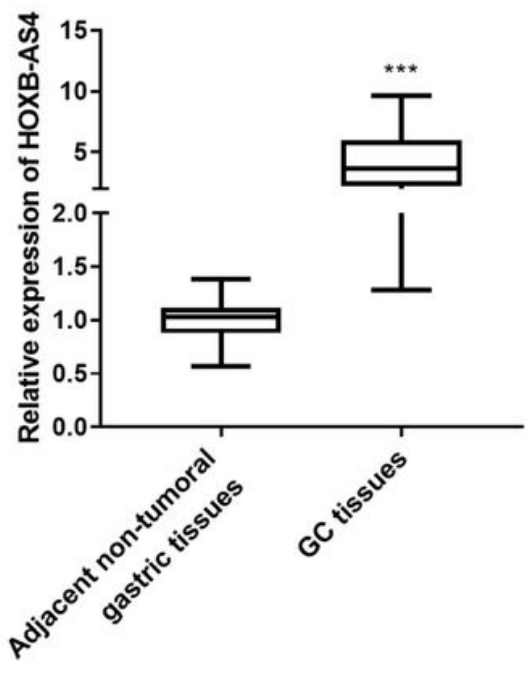

C

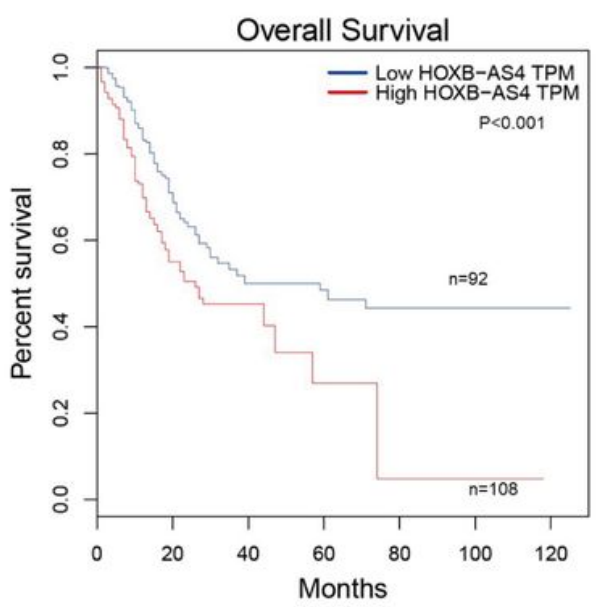

D

\section{Figure 1}


(A) LncRNA HOXB-AS4 is up-regulated in the patients' cohort of bladder urothelial carcinoma (BLCA), kidney renal papillary cell carcinoma (KIRP), stomach adenocarcinoma (STAD), uterine carcinosarcoma (UCS), and esophageal carcinoma (ESCA). (B) qRT-PCR verified the upregulation of HOXB-AS4 in GC tissues. (C) Kaplan-meier analysis showed overall survival in GC patients with HOXB-AS4 expression. (D) Expression of HOXB-AS4 in GES-1 cells and GC cancer cells was measured by qRT-PCR. Data are presented as mean \pm s. e. $m$. ${ }^{\star \star} p<0.01,{ }^{\star \star *} p<0.001$, compared to the control group.

A

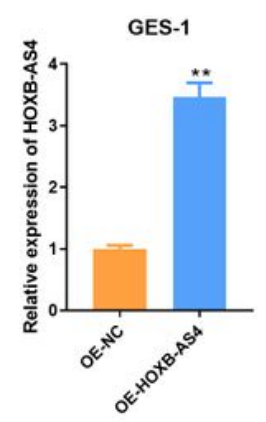

C

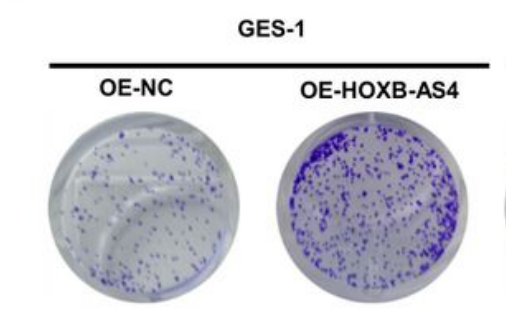

D

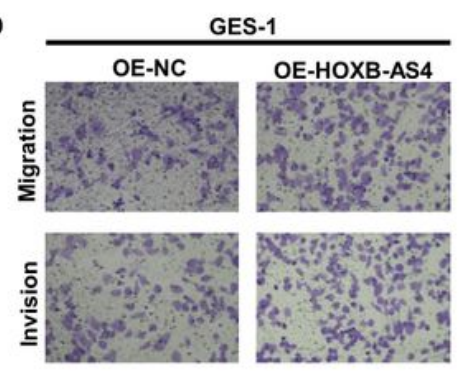

$E$

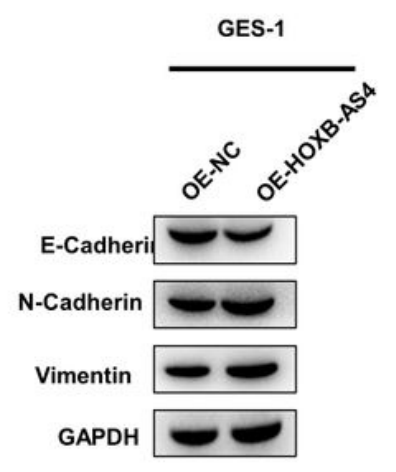

B

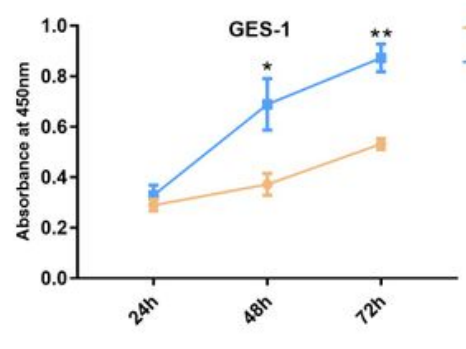

- OE-NC
- OE-HOXB-AS4
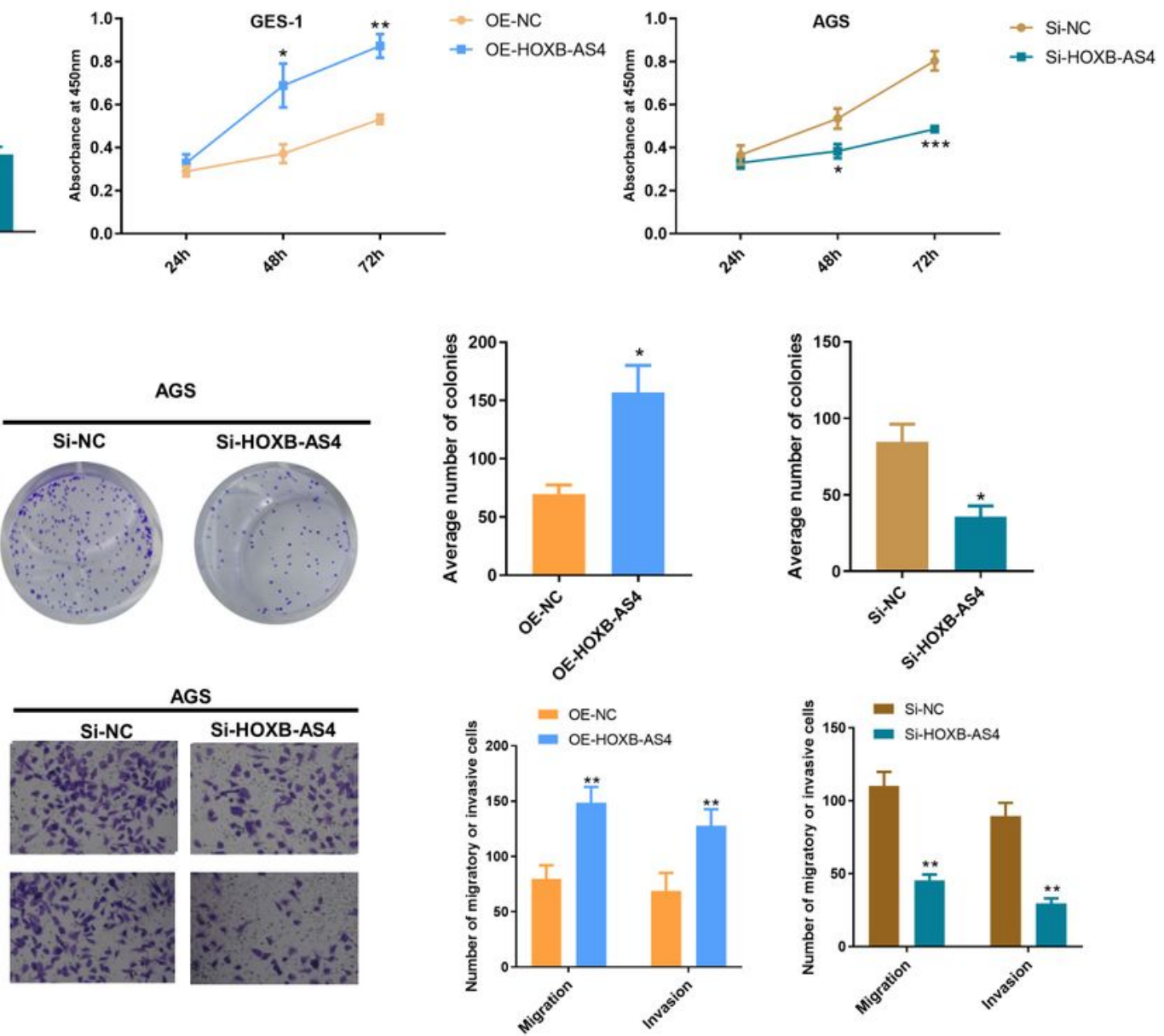

AGS

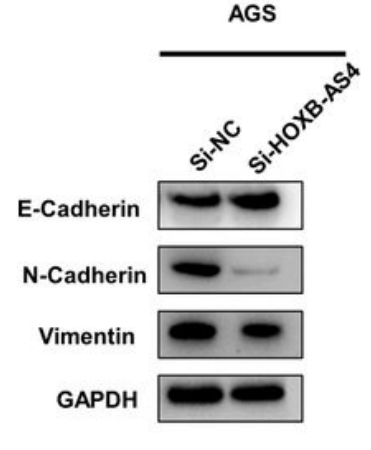

Figure 2 
HOXB-AS4 modulates GC cells proliferation, migration, invasion and EMT markers expression.

(A) qRT-PCR showed the transfection efficiency of overexpression or knockdown of HOXB-AS4 in GES-1 and AGS cells. (B) Cell viability of GES-1 overexpressed with HOXB-AS4 and AGS knocked down of HOXB$A S 4$ was detected by using MTT at 24,48 , and 72 hour. (C) Clone formation assay of GES-1 overexpressed with HOXB-AS4 and AGS knocked down of HOXB-AS4. (D) Transwell assay showed the migration and invasion of GES-1 overexpressed with HOXB-AS4 and AGS knocked down of HOXB-AS4. (E) Protein level of EMT markers in GES-1 cells overexpressed with HOXB-AS4 and AGS cells knocked down of $H O X B-A S 4$. $n=3$. Data are presented as mean \pm s. e. $m$. ${ }^{*} p<0.05,{ }^{*} p<0.01$, compared to the control group.

A

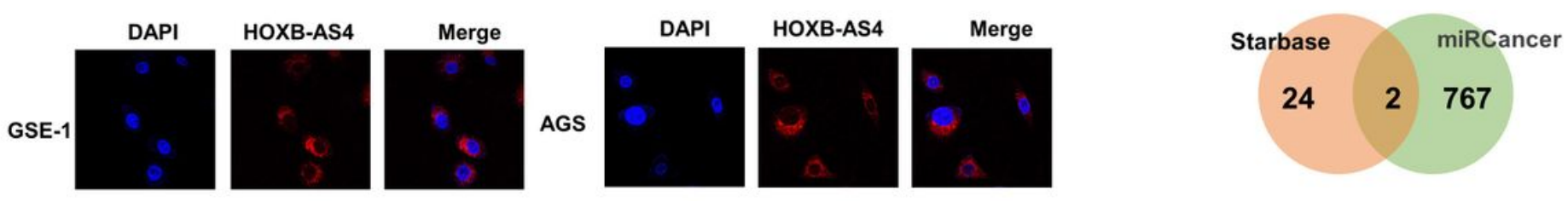

C

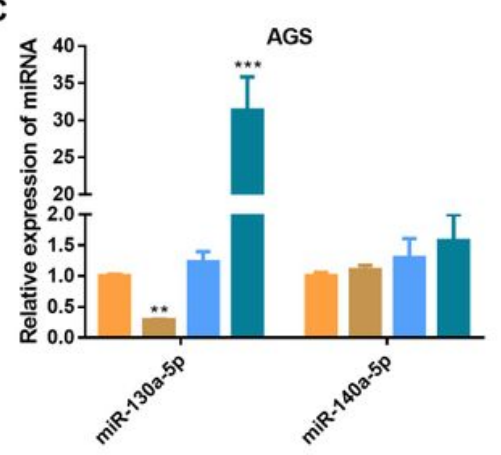

E
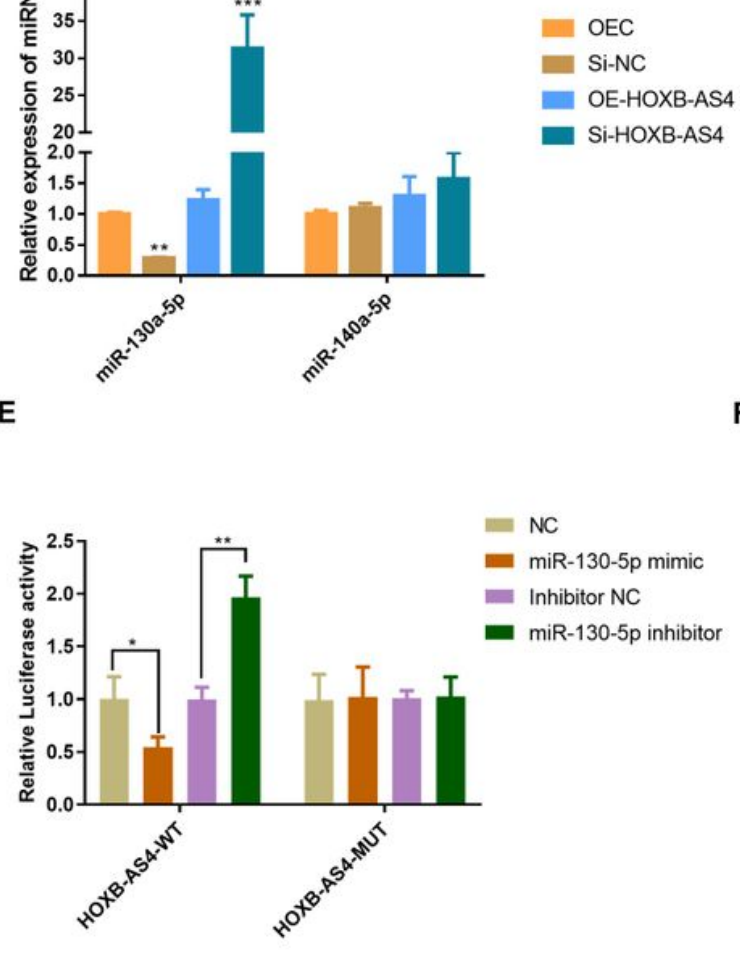

- Si-NC

OE-HOXB-AS4

- Si-HOXB-AS4

D

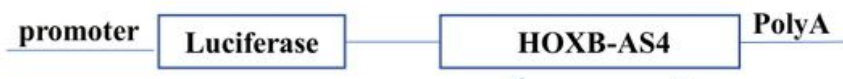

HOXB-AS4 WT 5' GTTTACTTtTTtTGAAAAAAgAgAaCtTGG 3' \|\|\|\|$\|$

hsa-miR-130a-5p 3' UCAUCGUGUUACACUUUUCUCG

HOXB-AS4 MUT 5' GTtTACTtTTtTtgaAtTTtGTGTACTTGG 3',

$\mathbf{F}$

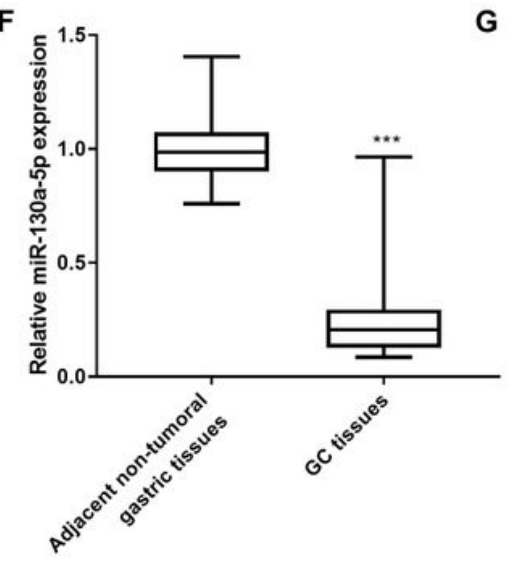

G

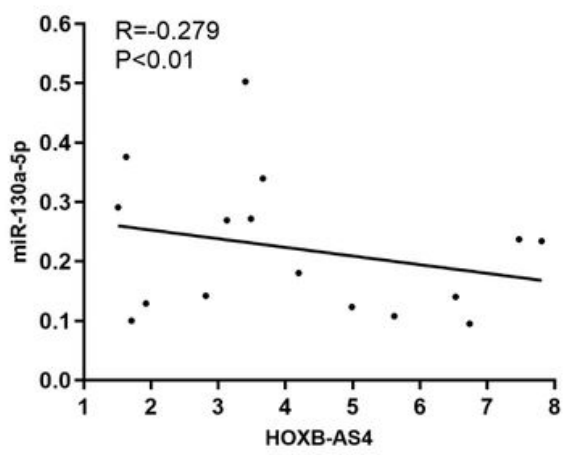

Figure 3

HOXB-AS4 acts as a sponge for miR-130a-5p.

(A) FISH analysis indicated subcellular location of HOXB-AS4 (red) in GSE-1 cells and AGS cells. (B) Schematic drawing of the screening intersection of the candidate miRNAs. (C) qRT-PCR was used to determine the level of miRNAs responded to overexpression or knockdown of HOXB-AS4. (D) The 
potential miRNA binding sites and the related mutant form were shown. (E) The luciferase reporter plasmid carrying wild type (WT) or mutant (MUT) HOXB-AS4 was co-transfected with miR-130a-5p mimic or inhibitor. Relative luciferase activity of the cells was measured. (F) miR-130a-5p in GC tissues was measured by qRT-PCR. (G) The negative correlation of HOXB-AS4 and miR-130a-5p expression in GC tissues $(n=16)$ was shown. $n=6$. Data are presented as mean \pm s. e. $m .{ }^{*} p<0.05,{ }^{*} p<0.01,{ }^{* \star *} p<0.001$, compared to the indicated control group.

A

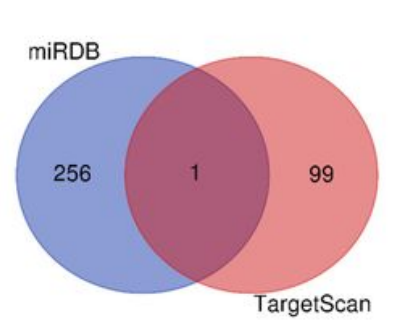

B

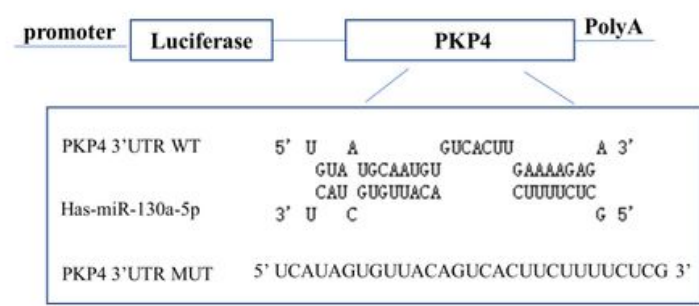

C

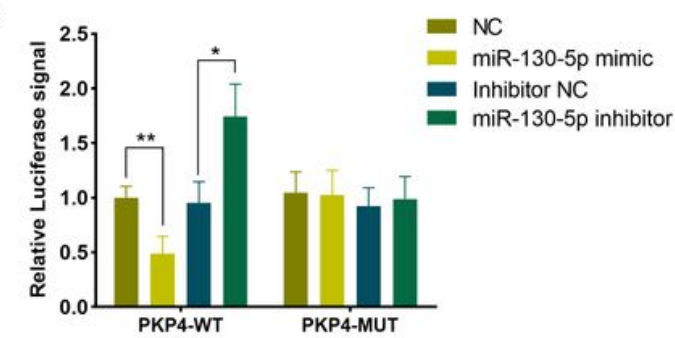

D
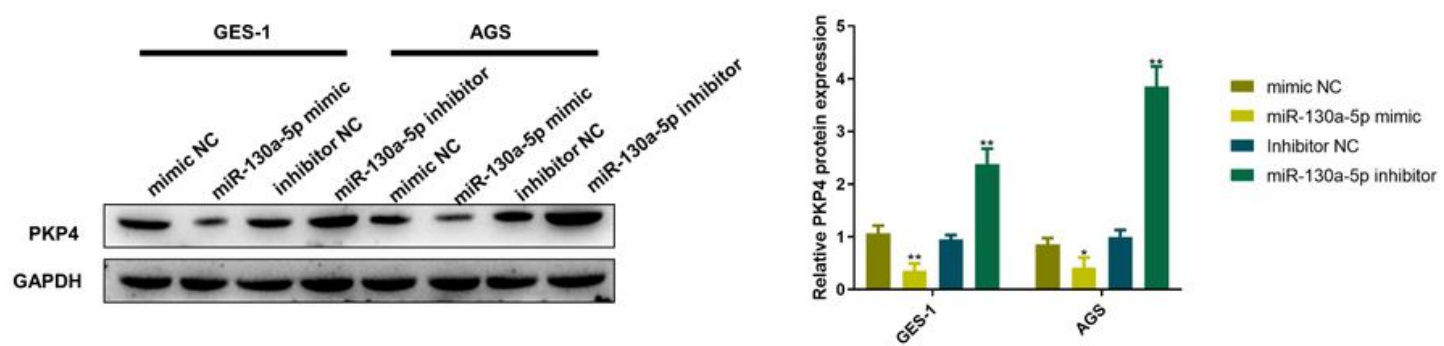

E
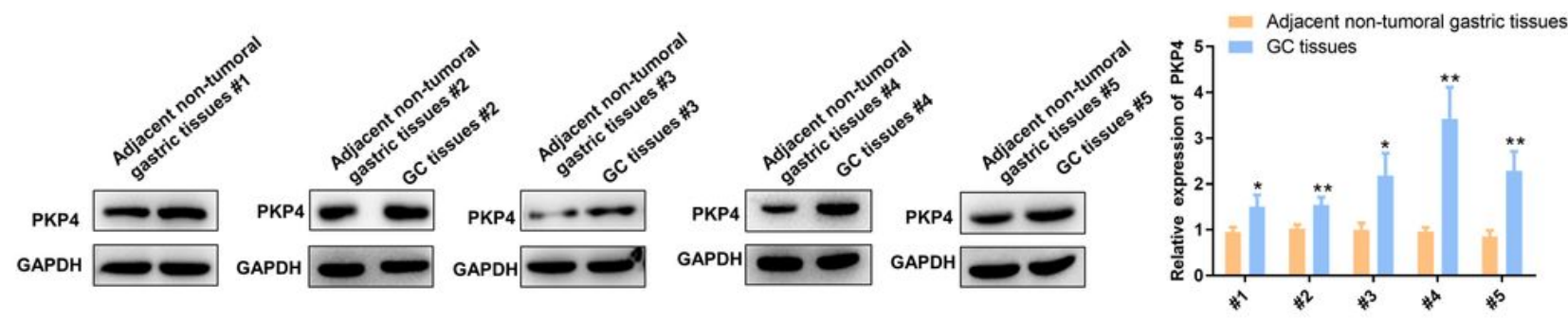

F
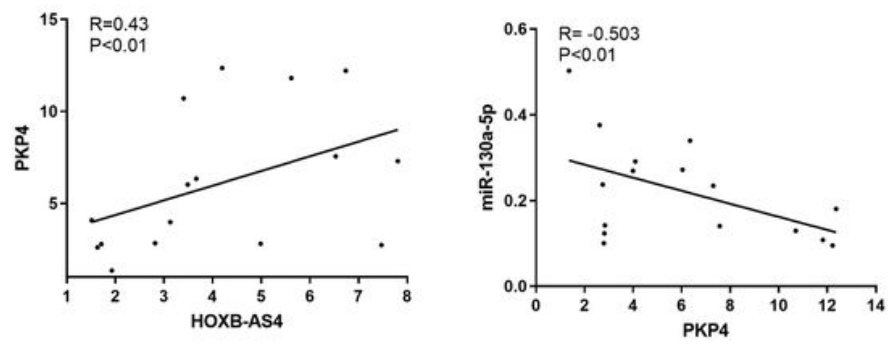

Figure 4

\section{PKP4 is a direct target of miR-130a-5p.}

(A) Schematic drawing of the screening intersection of the candidate target gene. (B) The potential miRNA binding sites and the related mutant form were shown. (C) The luciferase reporter plasmid carrying wild type (WT) or mutant (MUT) PKP4 was co-transfected with miR-130a-5p mimic or inhibitor. Relative luciferase activity of the cells was measured. (D) PKP4 expression in GES-1 and AGS-1 cells treated with miR-130a-5p mimic or inhibitor was measured by western blot. (E) Western blot was used to 
determine the PKP4 expression in GC tissues. (F) The positive correlation of PKP4 and HOXB-AS4 and the negative correlation of PKP4 and miR-130a-5p expression in GC tissues $(\mathrm{n}=16)$ was shown. Data are presented as mean \pm s. e. $m$. ${ }^{\star} p<0.05,{ }^{* \star} p<0.01$, compared to the indicated control group.

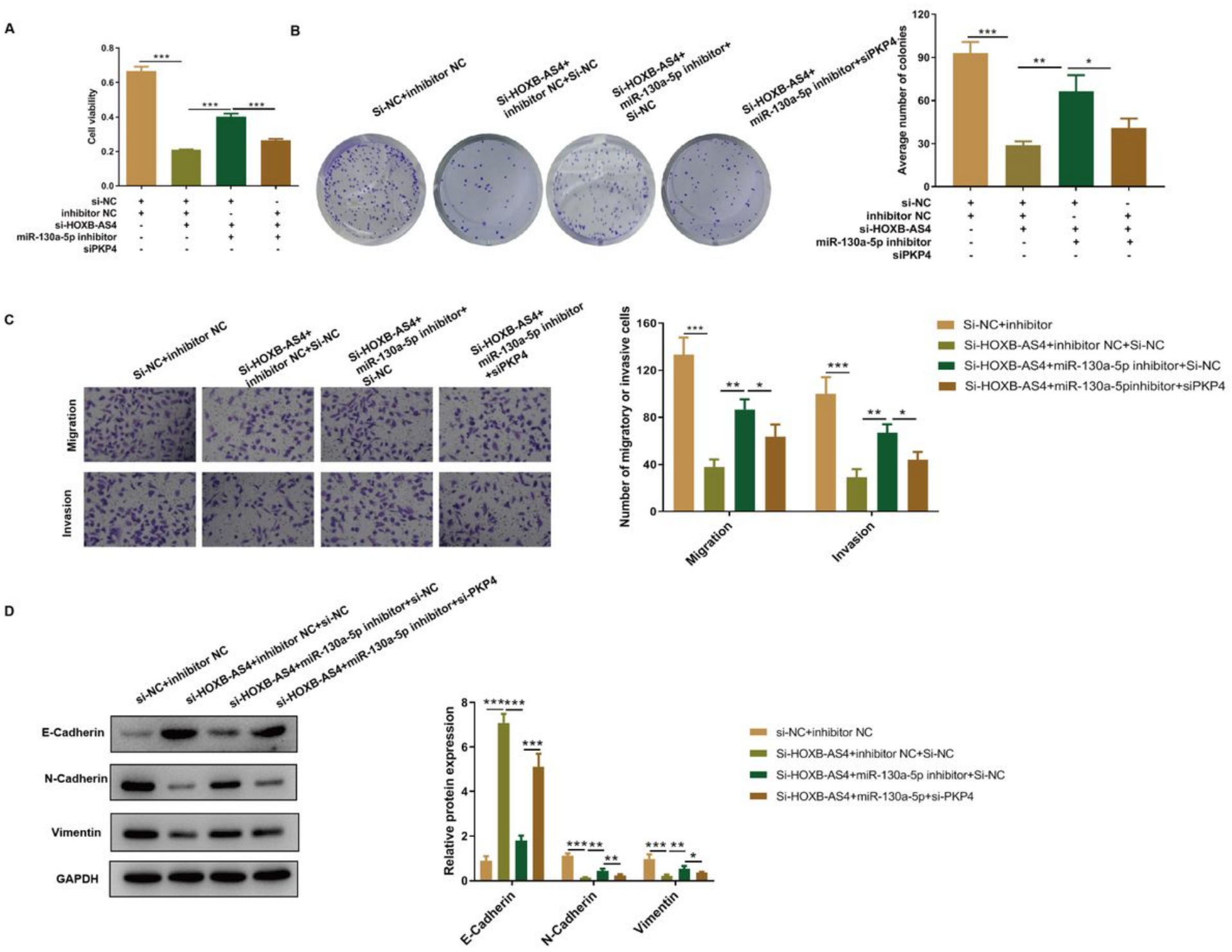

\section{Figure 5}

HOXB-AS4 modulates GC cells proliferation, migration, invasion and EMT markers expression via miR$130 \mathrm{a} /$ PKP4 axis.

AGS cells were knocked down of HOXB-AS4 singly, or with knockdown of miR-130a-5p or knockdown of PKP4 collectively. (A) Cell viability of AGS cells was determined by MTT. (B) Clone formation assay of AGS cells in the indicated group. (C) Migration and invasion of cells in the indicated group were measured by transwell assay. (D) Protein markers of EMT in AGS cells were detected by western blot. $n=6$. Data are presented as mean \pm s. e. $m .{ }^{*} p<0.05,{ }^{\star *} p<0.01,{ }^{* \star *} p<0.001$, compared to the indicated group. 
A

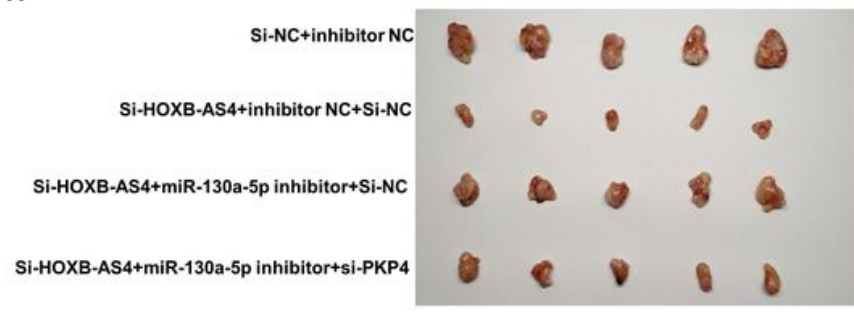

C

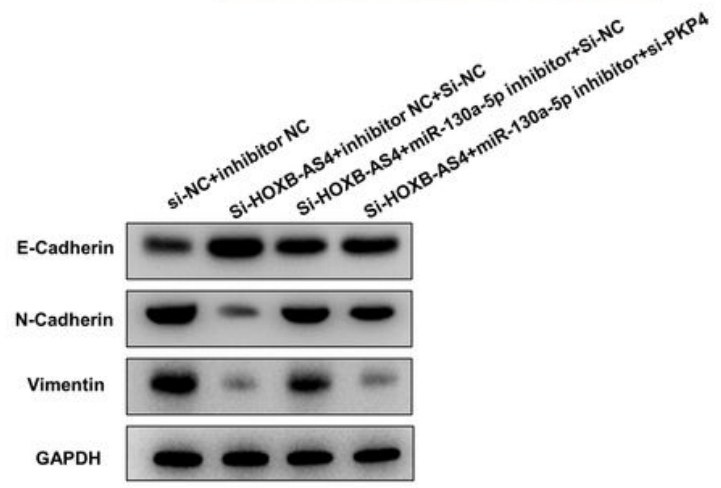

D
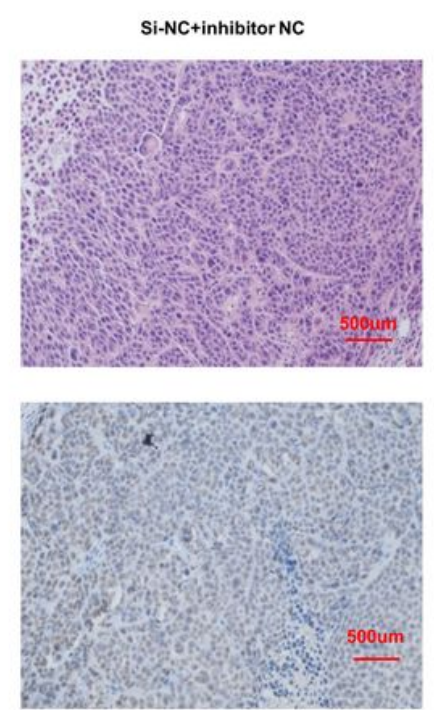
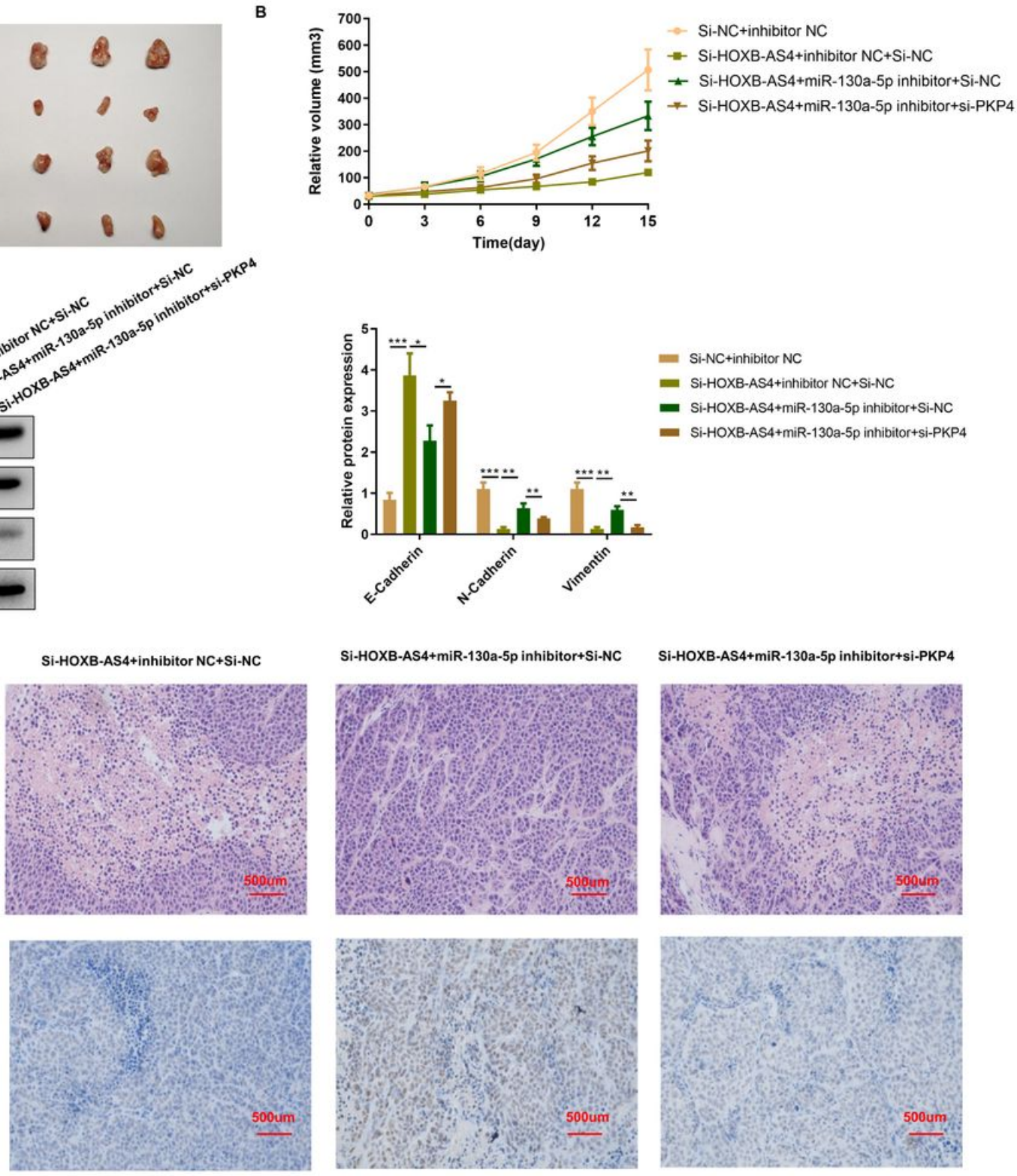

Si-HOXB-AS4+miR-130a-5p inhibitor+si-PKP4
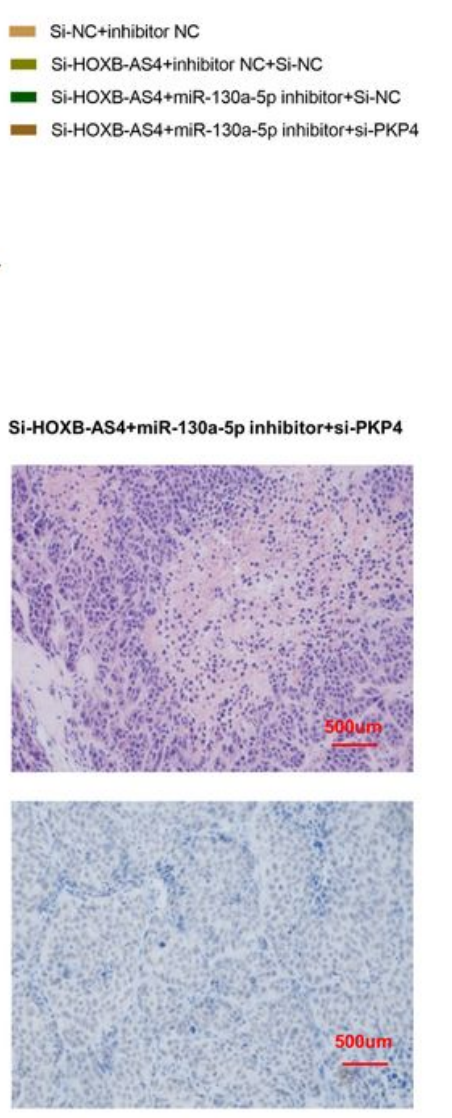

Figure 6

\section{Knockdown of HOXB-AS4 inhibits GC progression and modulates EMT markers expression in vivo.}

Mice were divided into four groups, with 10 mice in each group. miR-130a-5p antagomir $(80 \mathrm{mg} / \mathrm{kg}) \mathrm{was}$ administrated by tail injection. Knockdown of HOXB-AS4 or PKP4 was performed by using the related knockdown lentivirus $\left(4 \times 10^{7} \mathrm{IU} / \mathrm{mL}, 50 \mu \mathrm{L}\right)$ by intra tumoral injection. After 15 days, the mice were sacrificed and subjected to following experiments. (A) Pictures of the isolated tumors of the indicated group. (B) Change of tumor volume was recorded every three days. (C) Protein markers of EMT in tumors were detected by western blot. (D) H\&E staining of tumor tissues and IHC staining of PKP4 expression in tumor tissues were shown. $n=10$. Data are presented as mean \pm s. e. $m .{ }^{*} p<0.05,{ }^{* \star} p<0.01,{ }^{* \star *} p<0.001$, compared to the indicated group. 


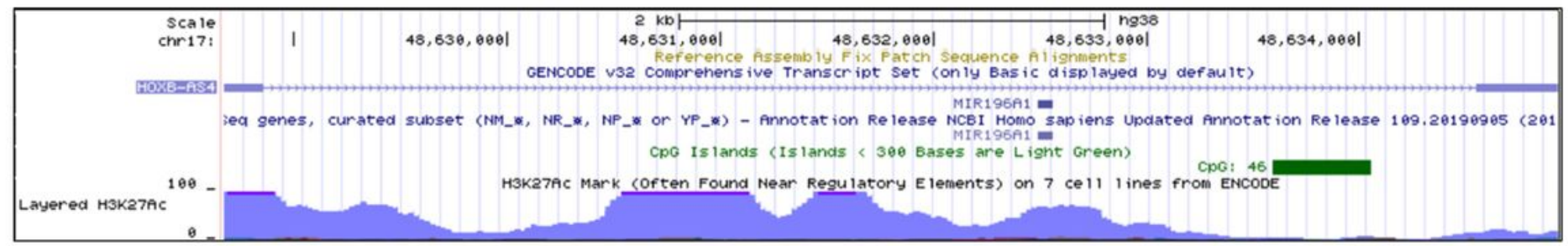

B

C
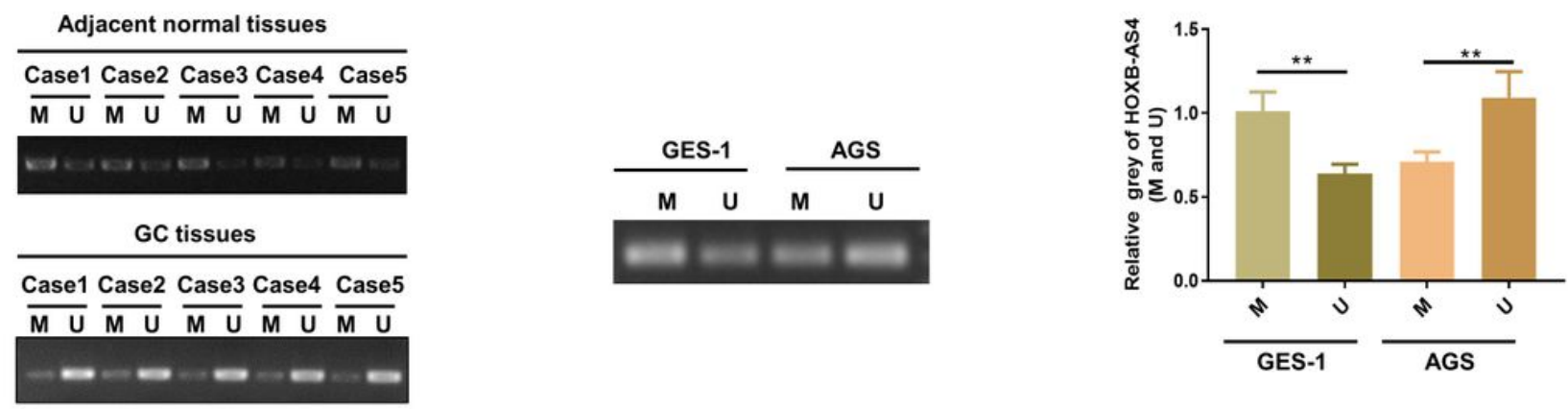

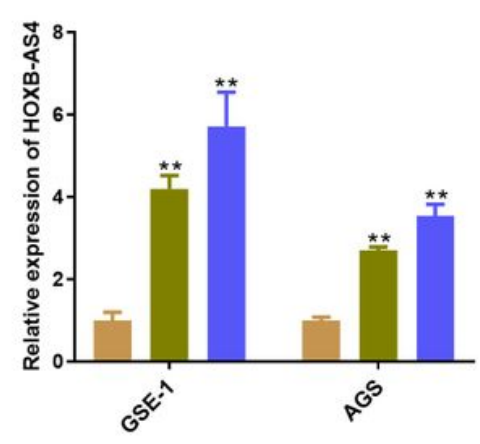

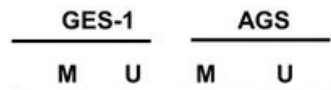

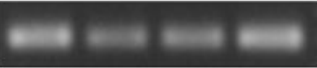

E

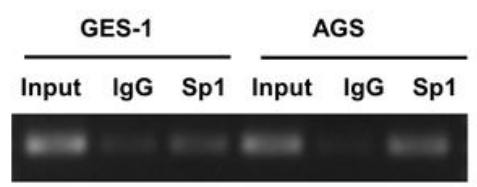

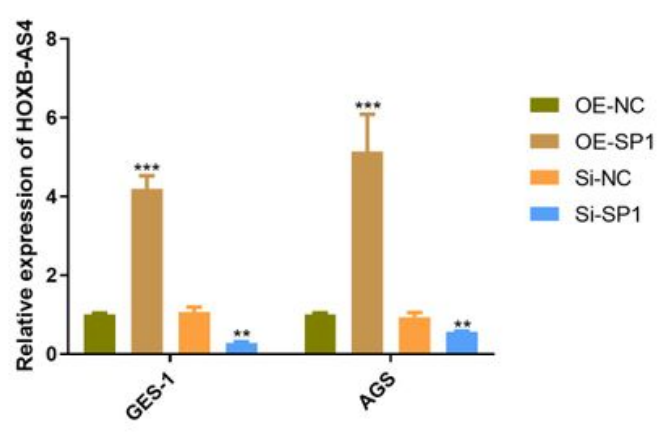

G
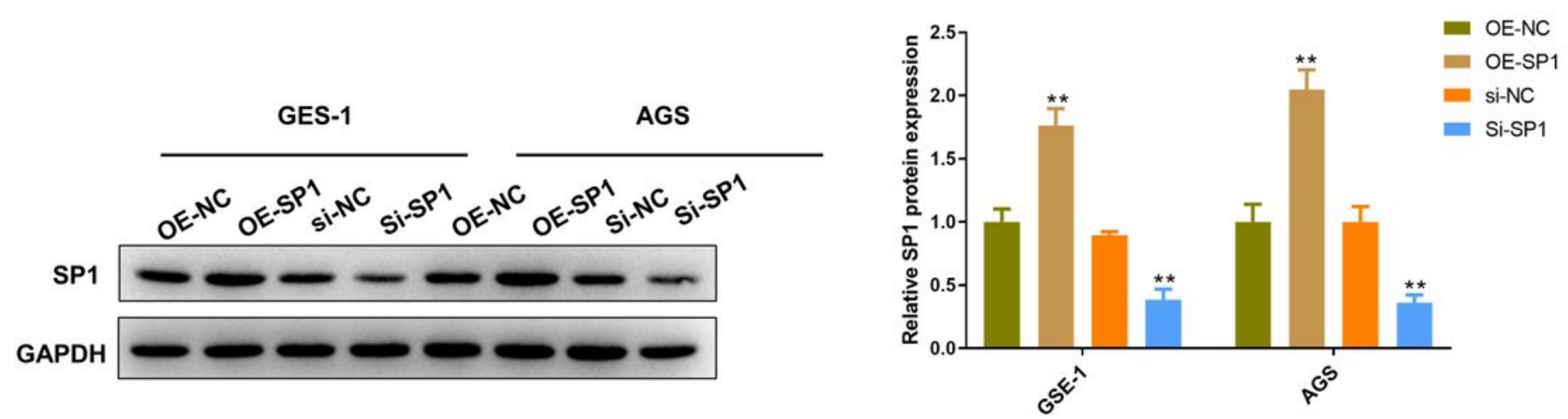

Figure 7

DNA methylation and SP1 participate in the regulation of the expression of HOXB-AS4.

(A) The UCSC Genome Bioinformatics Site (http://genome.ucsc.edu/) showed high enrichment of $\mathrm{H} 3 \mathrm{~K} 27 \mathrm{ac}$ at the promoter of HOXB-AS4. (B) MSP analysis was performed to examine methylation status of $\mathrm{CpG}$ island at the promoter region of $H O X B-A S 4$ in GC tissues and adjacent normal tissues. (B) MSP analysis was performed to examine methylation status of $\mathrm{CpG}$ island at the promoter region of $\mathrm{HOXB}$ - 
AS4 in GES-1 cells and AGS cells. (D) qRT-PCR analysis was used to detect the HOXB-AS4 level in GES-1 and AGS cells under the treatment with 5-azacytidine for $72 \mathrm{~h}$ and $120 \mathrm{~h}$. (E) ChIP analysis was used to detect the SP1 1 binding to the promoter region of HOXB-AS4. (F) HOXB-AS4 level in GES-1 and AGS cells overexpressed or knocked down with $S P 1$ was determined by qRT-PCR. (E) The transfection efficiency of $S P 1$ was verified by western blot. $\mathrm{n}=6$. Data are presented as mean \pm s. e. $m$. ${ }^{* \star} \mathrm{p}<0.01,{ }^{\star \star \star} \mathrm{p}<0.001$, compared to the indicated control group.

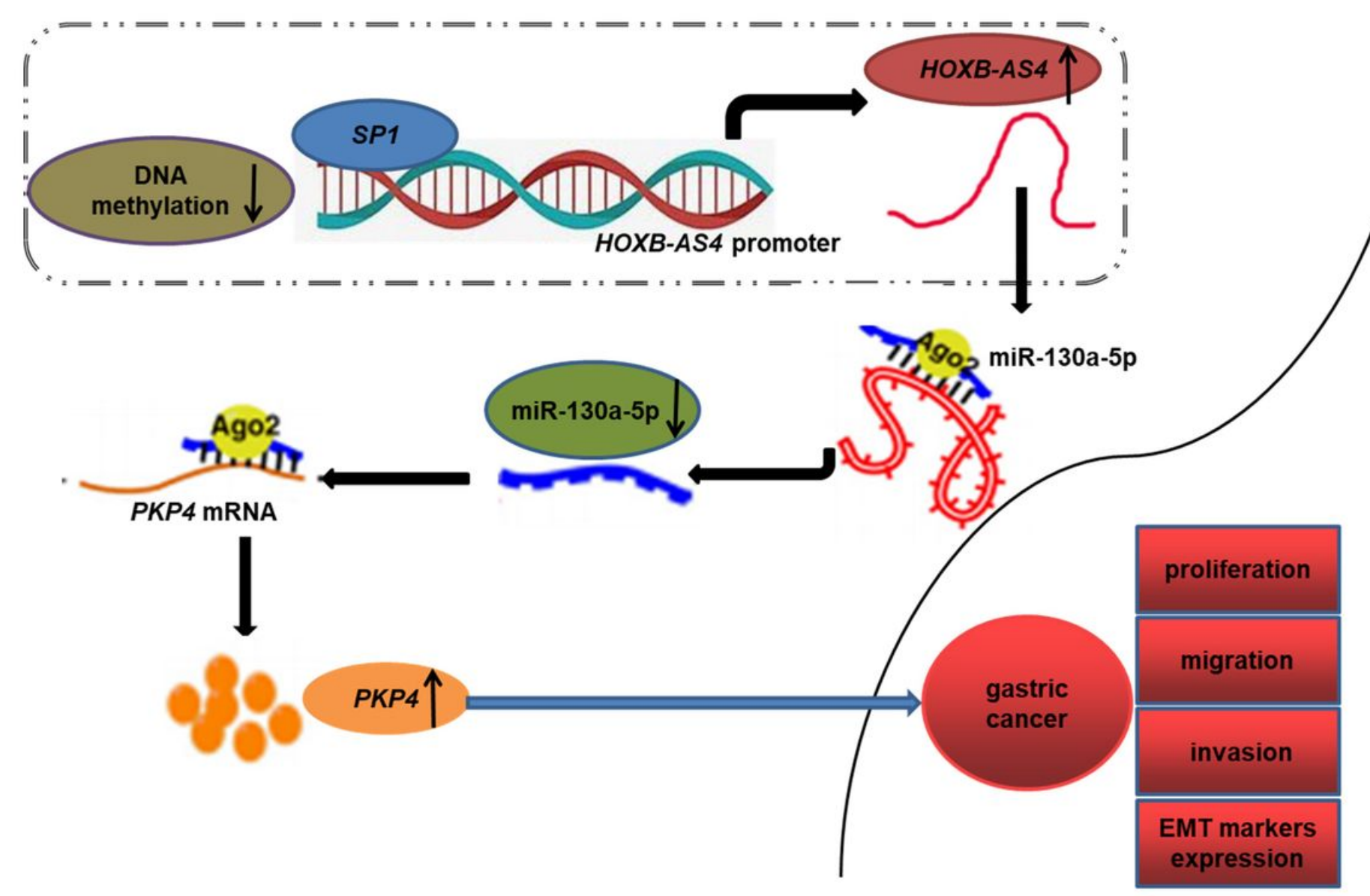

Figure 8

Proposed model in which HOXB-AS4 mediates the progression of GC.

\section{Supplementary Files}

This is a list of supplementary files associated with this preprint. Click to download.

- FigureS1.jpg

- Additionalfile1.doc 